\title{
Classification of Voltage Sags based on Multiway Principal Component Analysis and Case Based Reasoning ${ }^{\star}$
}

\author{
A. Khosravi* J. Melendez* M. Zapateiro* J. Colomer* \\ * Institut d'Informtica i Aplicacions, Universitat de Girona, Spain \\ (Tel: +3497241883; e-mail: quimmel@eia.udg.es).
}

\begin{abstract}
:
Impact of a bad power quality on customers has motivated the development of a classification strategies to identify sags (short duration voltage falls in the power system) and assist the location of its origin. The paper proposes a new method to classify voltage sags registered in distribution substations based on the combination of statistical and reasoning methods. The goal is to associate a sag waveform with its origin (Medium Voltage -MV- or High Voltage -HV-) in the network. Multiway Principal Component Analysis (MPCA) is used as dimension reduction strategy whereas Case Based Reasoning (CBR) is applied in this projection space to retrieve events previously registered and diagnosed. Capability of the proposed method has been demonstrated with data gathered in five different substations of the power system in Catalonia (Spain).
\end{abstract}

\section{INTRODUCTION}

Nowadays, the electricity dependence of industries, commerce and services has provoked the regulation of power quality. The objective is to reduce damages or misbehaviors to consumer devices and/or processes. From generators to customers, the voltage waveform can suffer alterations that affect quality. Origin of such alterations can be associated to multiple agents involving the normal operation of the network (firing protections, switching taps and lines,etc.), external agents (animals, vegetation, weather,etc.) or due to the operation of specific loads (motors, capacitor banks,etc.). This alteration of the sinusoidal wave is usually transmitted to the electrical system [2] and the responsibility of possible damages caused to customers is usually assigned to the utilities. In order to delimitate its responsibility an at same time improve system knowledge and management, the utilities are monitoring the networks and promote the development of intelligent strategies to improve the capabilities of such a monitoring systems. Sag coordination charts are used with this proposal to classify registered sags in several categories according to its depth and duration. In a similar way power quality surveys are created with data from multiple monitors gathered during long periods of time (one or plus years) to evaluate the quality of power systems. Other strategies try to improve power quality monitoring by focusing on the accurate analysis of disturbances. Wavelet decomposition [8, 11, 15] or attribute based description of disturbances [12] has been used with this aim.

Principal Component Analysis (PCA) and, more recently, its extension Multiway PCA (MPCA) have been used for fault diagnosis and monitoring of complex processes in


y Ciencia of the Spanish Governmet (DPI2006-09370) and the utility ENDESA DISTRIBUCION (PTR95.1020.OP)
}

the industry by exploiting existing historical data [16]. Dimensionality reduction by preserving the variability of original data is in the core of such a techniques. Similarly, Case Based Reasoning (CBR) has been demonstrated to be an efficient technique for problem solving based on the reuse of previous experiences. A new problem is matched against a set of cases stored in the case base and the most similar ones are retrieved and reused, after a revision stage, to solve the former [1]. Both techniques, MPCA and CBR, have been combined to determine the origin of sags registered in distribution substations. Cases have been defined in the projection space resulting from applying MPCA models to rms current and voltage waveforms of registered sags.

The article is organized in VI main sections. In section II a complete description of the problem to be solved and specific goals are presented. A brief review of the MPCA and CBR concepts is given in section III. Section IV is devoted to present the proposed methodology involving MPCA and CBR. Finally, Section V and VI present the main results and conclusions respectively.

\section{PROBLEM OVERVIEW AND OBJECTIVES}

Several types of disturbances are known to cause problems in the performance of electric equipment. Swells, sags, harmonic distortions and momentary interruptions are the most common. Among them, voltage sags are the most significant according to their severity, number of occurrences per year and effects on customers. A sag is a reduction in the voltage, usually below $90 \%$ of the nominal voltage level, lasting from a few cycles up to a few seconds affecting one, two or three phases. The evolution of RMS value of sag is represented in Fig.1.

Their origin can be either in the transmission or the distribution system and they propagate through the network 



(b)

Fig. 1. Voltage Sag: evolution of rms voltage and current

to customers. Typical causes are short circuits, lightning during storms, defective wiring, grounding problems or the connection of large loads. Based on the sag duration and its magnitude, several works have been performed to characterize and classify sags.

The objective in this work is to evaluate sags waveform and decide whether a voltage sag has occurred in the Medium Voltage (MV) system or in the High Voltage (HV) system of the electrical network. A combination of MPCA and CBR has been used with this purpose working on data gathered in real substations by using power quality monitors.

\section{THEORETICAL BACKGROUND}

In this section, we present a summary of both MPCA and CBR which have been used for developing the proposed hybrid method in this paper.

\subsection{Principal Component Analysis (PCA)}

The most extended tool for data compression and information extraction is the Principal Component Analysis (PCA). It finds combinations of variables or factors describing major trends in a data set [3]. That is, PCA is concerned with explaining the variance-covariance structure through a few linear combinations of the original variables. Its general objectives are data reduction and interpretation [5]. Multivariate data can be organized in variables and samples or observations per variable $(\mathrm{X})$. The covariance matrix, S, of X determines a set of orthogonal vectors, called loading vectors:

$$
S=\frac{1}{n-1} X^{T} X
$$

which are calculated by solving the stationary points of the optimization problem [14]:

$$
\max _{v \neq 0} \frac{v^{T} X^{T} X v}{v^{T} v}
$$

This can be computed via the singular value decomposition for obtaining a diagonal matrix $\Sigma \varepsilon R^{m \times m}$ which contains the nonnegative real eigenvalues of decreasing magnitude. Selecting the columns of the loading matrix $P \varepsilon R^{m \times m}$ to correspond to the loading vectors associated with the first a singular values, the projections of the observations in $X$ onto the lower dimensional space are contained in the score matrix:

$$
T=X P^{T}
$$

and the projection of $T$ back onto the $m$-dimensional observation space:

$$
\hat{X}=T P^{T}
$$

The difference between $X$ and $\hat{X}$ is the residual matrix $E$ [14]. Thus, the first principal component is defined as the linear combination $t_{1}=X p_{1}$ which has maximum variance subject to $\left|p_{1}\right|=1$. The second principal component is the linear combination defined by $t_{2}=X p_{2}$ which has the next greatest variance subject to $\left|p_{2}\right|=1$ and subject to the condition that it is uncorrelated with (orthogonal to) the first principal component $\left(t_{1}\right)$. Up to $N$ principal components are similarly defined [6]. The principal components represent the selection of a new coordinate system obtained by rotating the original variables and projecting them onto the reduced space defined by the first few principal components, where the data are described adequately and in a simpler and more meaningful way. The principal components are ordered such that the first one describes the largest amount of variation in the data, the second one the second largest amount of variation, and so on [13]. By retaining only the first $N$ principal components, the $X$ matrix is approximated by (5) [9]. PCA can be mathematically expressed as follows [6]:

$$
X=\sum_{j=1}^{N} t_{j} p_{j}^{T}+E
$$

where $N$ is the number of principal components. Two complementary multivariate control charts are required for process monitoring using projection methods: $T^{2}$ and $Q$-statistic. Multivariate control charts based on $T^{2}$ can be plotted based on the first $N$ principal components as follows [9]:

$$
T^{2}=\sum_{j=1}^{N} \frac{t_{j}^{2}}{S_{t_{j}}^{2}}
$$

Where $S_{t_{j}}^{2}$ is the estimated variance of $t_{j}$. This control chart will only detect variation in the plane of the first $N$ principal components which are greater than what can be explained by the common-cause variations. When a new type of special event occurs which was not present in the in-control data used to build the PCA model, the new observations will move off the plane. This type of event can be detected by computing the Q-statistic or Squared Prediction Error (SPE) of the residual for new observations. It is defined as [9] [20]:

$$
Q_{X}=\sum_{j=1}^{N}\left(x_{j}-\hat{x}_{j, \text { new }}\right)
$$

where $\hat{X}_{n e w}$ is computed from the reference PCA model. Normally, $Q$-statistic is much more sensitive than $T^{2}$. It 
is due to this fact that $Q$ is very small and therefore any minor change in the system characteristics will be observable. $T^{2}$ has a great variance and therefore requires a great change in the system characteristic for it to be detectable.

\subsection{Multiway Principal Component Analysis (MPCA)}

An extension of PCA models specially conceived for working with batch process is MPCA (Multiway PCA). In this approach each batch is represented by a matrix containing the evolution of sensors during the batch execution (each column represents one variable and each row a time instant during the batch execution). Consequently, the initial dataset or observations matrix, X, built from a set of I batches (or observations) becomes a three dimensional matrix (IxJxK) containing I observations or batches each one described by $\mathrm{J}$ variables sampled during $\mathrm{K}$ consecutive instants during the batch execution. In order to capture the correlation in the direction of both variables and time, this three dimensional matrix is unfolded in the direction of batch resulting a two dimensional matrix (IxJK). This new matrix (2D) contains the same information as the original one but reorganised resulting in one row all the information from one single batch. In other words, one row of the unfolded matrix contains the sequence of samples of each variable registered during a batch $(\mathrm{J}$ times $\mathrm{K})$. The PCA methodology is then applied to this new matrix in order to capture relationships among variables and samples extracted from the set of batches (I) [20] [19]. A parallelism between batch concept and sags have been performed in this work: Registers of sags contain waveforms of three voltage phases and three line currents $(\mathrm{J}=6)$, gathered during a fix duration time ( 0.78 seconds) and sampled at $6400 \mathrm{~Hz}(\mathrm{~K}=4992)$. The number of sags registered in a substation (see Table 1) used to built the model corresponds to I dimension.

All the equipments used in the experiment have been configured to start the register of sags two periods of the waveform before its detection and the detection threshold has been configured to be $10 \%$ below the nominal value rms voltage.

\subsection{Case Based Reasoning (CBR)}

CBR is a reasoning methodology for reusing experiences. Simply, CBR is based on the following assumptions: if the source case is similar to the query case, its solution should be similar as well and therefore transferable, perhaps with modifications [4] [18]. In other words, we can reuse solution of a similar problem to solve an actual problem. CBR completes those tasks in a four-step cycle $(4 R$ cycle $)$. Experiences about a problem and its solution are continuously coded and Retained as cases for its further Reuse. Some of these cases will be Retrieved for finding the best solution to a new one, according to a similarity criteria. Afterwards, through an adaptation procedure, we apply the Revised solution to the case, and finally in the last step, the solved situation (problem and solution) can be retained in the case base, after an evaluation, as a solved case for a possible future reuse [10]. Consequently, the further the time it runs, the better the decisions are made. Interested readers can find CBR foundations and a

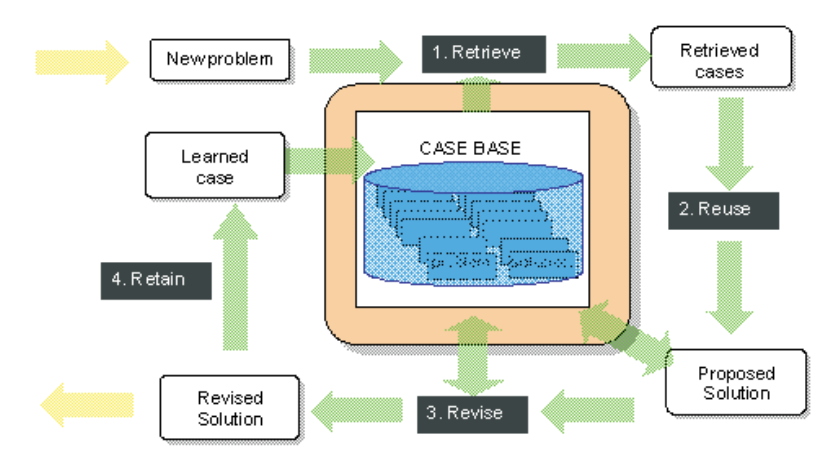

Fig. 2. CBR cycle

detailed description of this methodology based on the $4 R$ in [1] and [7].

\section{METHODOLOGICAL ASPECTS}

In this section, we explain how MPCA is used for classification of electrical sags according to its origin in High Voltage (HV) and Medium Voltage (MV) lines using a statistical criterion based on the $T^{2}$-Hotelling and $Q$-residual statistics. Afterwards, results are improved based on the concept of CBR in the projection space described by principal components. The following steps for the construction of the dataset matrix, $\mathrm{X}$, have been considered:

(1) Preprocessing: Instantaneous rms value for each variable (three voltages and three currents) is computed. FFT in a one period (20 msec. $-50 \mathrm{~Hz}-)$ sliding window is used to isolate the estimate the magnitude of nominal frequency $(50 \mathrm{~Hz})$ during the sag. Preprocessing finishes with autoscaling (zero mean centered and unit variance) transformation applied to the variables in order to reduce overweight of voltage variables towards current due to its bigger magnitude.

(2) Dataset organisation and labelling: A two dimensional matrix is built containing in each row information related to one single sag. Each row contains as many elements as number of variables times samples per variable (JK). For each substation the number of sags available (number of rows) is different (see Table 1). The original dataset also contains information about the origin of these sags and have been labelled as MV (Medium Voltage) or HV (High voltage) to identify downstream or upstream origin of disturbances.

(3) Data cleaning: A MPCA analysis has been performed, before building the final model for classification, with the original dataset organised in two subsets containing only HV or MV sags. These models have been used to identify outliers and registers containing nonnormal behaviours. $Q$ and $T^{2}$ statistics have been used with a confidence level of 0.95 to delimitate them (See table 1). The majority of these abnormal registers do not correspond to sags; they can be caused by other disturbances as interruptions (deeper than $90 \%$ ), subvoltages (longer than a sag) or transients (shorter than sags) or they can contain only part of a sag (bad registers). The same threshold will be applied to new sags before applying the classification method. Those that overpass the threshold are considered unclassified. 
The resulting data set, $\mathrm{X}$, has been used in training and testing purposes according to the following considerations:

- Training and Test data sets: The cleaned data sets containing HV (idem for MV) has been randomly split into two subsets: training $(75 \%)$ and test $(25 \%)$ subsets.

- Building MPCA models and validation: The HV (MV) training data subset (75\% of the total) has been used to create an HV (MV) MPCA model. In the validation step testing subset $(25 \%)$ has been used. 4 -fold cross validation has been applied in evaluating the results.

- Model Exploitation: The test subset has been spread using registers from the other class MV (HV). The projection of this new testing subset allows to evaluate how the model performs with sags from different class.

Following these stages, the traditional statistical method derived from MPCA technique has been applied and improved using CBR methodology in the decision step for locating the origin of new sags.

\subsection{Traditional statistical method}

The test set is projected onto both $\mathrm{HV}$ and MV MPCA models to classify them according to its origin or class. A decision is made based on the following criteria:

(1) A test batch projected onto the HV (MV) MPCA model will be considered as an HV (MV) batch if the $T^{2}$ and $Q$ statistics of its projection lie inside the region delimited by $Q$ and $T^{2}$ of the model. Otherwise, it will assigned to the other class, MV (HV) by this model HV (MV).

(2) If the conclusion after the projection of the test sag on both models $\mathrm{HV}$ and MV is the same, there is a decision; otherwise no decision can be made.

Results using this approach are summarised in Table II and commented in the next section.

\subsection{Hybrid MPCA-CBR method for fault location}

In this subsection, we develop a CBR-based classifier implemented on the projection space obtained from the MPCA models. The proposed method after the projection of test data onto both HV and MV models, continues with the following stages (See Fig.3):

(1) Distance calculation: On the $T^{2}-Q$ plane, the euclidean distance (principal component space is orthogonal) between the projection of each test sag and the training sags in the transformed space of the $\mathrm{HV}$ and MV MPCA models is calculated.

(2) Sorting: Distances are reordered in an ascending way to identify the sagas closer to the tested one.

(3) Distance-based decision $(C B R)$ : The dominant class of the majority of the $K$-Nearest neighbors inside a surrounded space determines the class of the tested sag; i.e. if most of the neighbors are $\mathrm{HV}(\mathrm{MV})$, the test sag will be classified HV (MV).

The number of nearest neighbors $(K)$ is chosen to be an odd number in order to avoid draw results. Therefore,

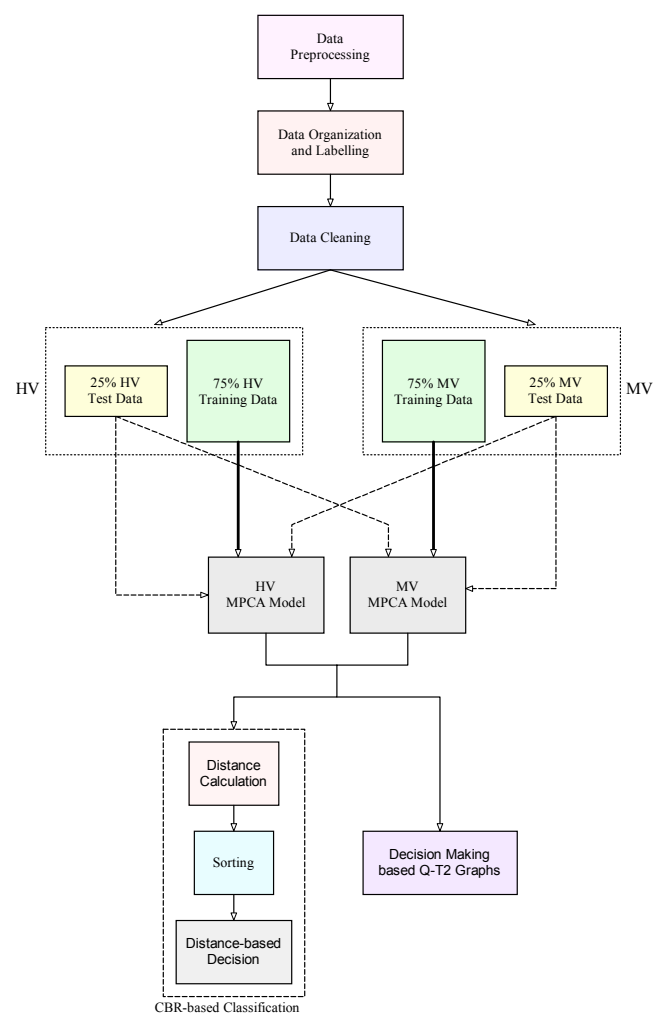

Fig. 3. Schematic of fault classification method.

always we will be able to make a decision. The surrounded space of each new (test) sag on the two-dimensional $T^{2}-$ $Q$ plane is a circle. The optimal values for $K$ and the circle radius have been determined through solving an optimization problem in this two dimensional space which leads to the maximum classification rate. Fig 4 and Fig 5 represent this dependency for MV and HV sags. We would like to highlight at this point that using autoscaling method for scaling $\mathrm{HV}$ and MV data makes reasonably possible that we calculate the distance between different sags without any concern about the voltage or currents amplitudes and their effects on scores in $\mathrm{HV}$ and $\mathrm{MV}$ MPCA models. In fact, not or using other scaling methods will lead to completely wrong decisions in CBR due to the big differences between voltages and currents of the $\mathrm{HV}$ and MV lines. Through this method, we bring them to one scale and can manipulate them in a similar method.

\section{FAULT LOCATION AND RESULTS}

The proposed method has been tested using sets of data taken from five electrical substations located in Catalonia, Spain. The data consists of preprocessed records of the three phase voltages and three currents in a number that depends on the substation as the two first columns in Table 1 summarises.

The first step was to generate the MPCA models for the $\mathrm{HV}$ and MV data. The data was auto-scaled in order to avoid problems dervide of different magnitude of variables. The confidence limit was set on $95 \%$ and the number of remaining sags, after the removal, is shown also in Table 1. These remaining data was used to build and test the 
Table 1. Number of sags per substation before and after the removal of non characteristic batches.

\begin{tabular}{ccccc}
\hline \hline & \multicolumn{2}{c}{ Total } & \multicolumn{2}{c}{ Remaining } \\
\hline Substation & HV & MV & HV & MV \\
\hline A & 77 & 69 & 62 & 53 \\
B & 69 & 75 & 60 & 56 \\
C & 75 & 83 & 63 & 65 \\
D & 59 & 308 & 52 & 245 \\
E & 142 & 101 & 109 & 77 \\
\hline
\end{tabular}

Table 2. Percentage rate of correct classification of sags using the traditional MPCA statistical method.

\begin{tabular}{ccccccc}
\hline \hline & & \multicolumn{5}{c}{ Substation } \\
\hline Class & Model & A & B & C & D & E \\
\hline HV & 1 & 33.33 & 33.33 & 60 & 76.92 & 3.70 \\
& 2 & 60 & 66.67 & 66.67 & 30.77 & 22.22 \\
& 3 & 40 & 40 & 53.33 & 30.77 & 37.04 \\
& 4 & 40 & 73.33 & 73.33 & 46.15 & 14.81 \\
\hline MV & Mean & 43 & 53 & 63 & 46 & 19 \\
& 1 & 61.54 & 50 & 62.50 & 88.52 & 57.89 \\
& 2 & 53.85 & 57.14 & 43.75 & 85.25 & 68.42 \\
& 3 & 69.23 & 64.29 & 56.25 & 73.77 & 52.63 \\
& 4 & 38.46 & 71.43 & 81.25 & 65.57 & 47.37 \\
\hline & Mean & 55.77 & 60.71 & 60.94 & 78.28 & 56.58 \\
\hline
\end{tabular}

models. A cross validation technique was used to create the training and test sets. Thus, the datasets were divided into 4 subsets; one of the subsets was used as a test set and the remaining data were used to build a model. This way, 4 different models could be built and tested for each class (HV and MV).

Provided the final data to work with, the MPCA models for both classes were built. The confidence limit was set again on $95 \%$ and the number of principal components used was 10 capturing a significant percent of total variance (around 90\%). Once obtained the HV and MV models, test sags were projected onto both models and, according to previous criteria, a sag has been considered to be correctly classified when it is assigned to the same class when projected to both models. The rates of correct classification following this criterion are summarized in Table 2 resulting in a poor success ratio.

Table 2 shows that HV class sags are hardly correctly classified while MV class sags have a better, though still low, rate of classification. Then, the CBR technique was applied on the projected data to improve the results. First of all, a nearest neighbour criteria was fixed in order to identify the best value of $K$ (number of neighbours). As explained earlier, the optimal values for $K$ and the circle radius are determined through solving an optimization problem in two dimensions. The well known Euclidean distance definition was used to find the nearest neighbors. Several simulations were run; the number of neighbors was varied from 3 to 11 and the circle radius was varied from 100 to 5000. Following this methodology, the circle radius used to classify the test batches was 1250 and the number of neighbours $(\mathrm{K})$ was set on 5 . This selection is based on the average values obtained when applying the 4 -fold cross validation (represented in the Fig. 4 and Fig.5).

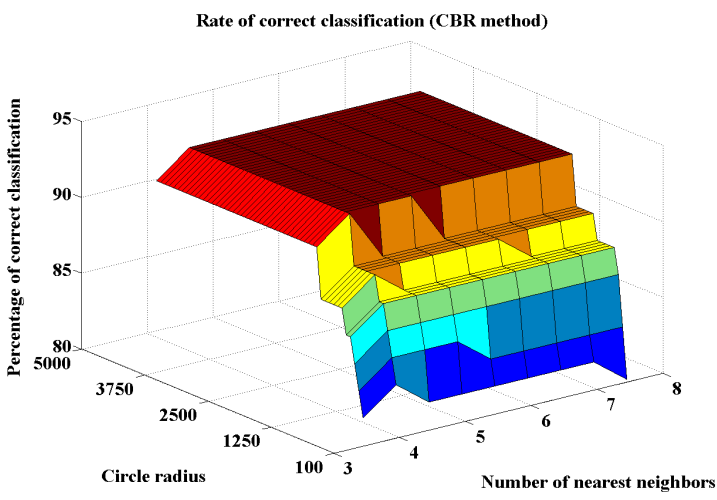

Fig. 4. Percentage of correct classification of HV sags using the CBR method.

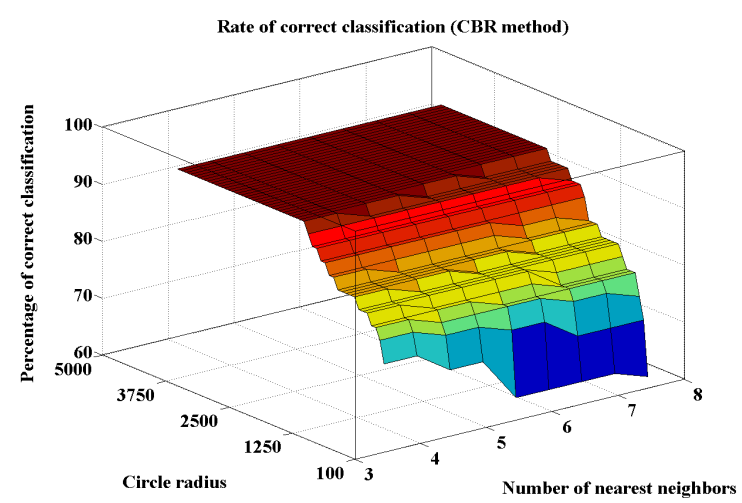

Fig. 5. Percentage of correct classification of MV sags using the CBR method.

Table 3. Percentage rate of correct classification of sags using the proposed method based on CBR.

\begin{tabular}{ccccccc}
\hline \hline & & \multicolumn{5}{c}{ Substation } \\
\hline Class & Model & A & B & C & D & E \\
\hline HV & 1 & 93.33 & 100 & 100 & 100 & 74.07 \\
& 2 & 100 & 100 & 86.67 & 100 & 70.37 \\
& 3 & 86.67 & 73.33 & 66.67 & 100 & 88.89 \\
& 4 & 100 & 100 & 93.33 & 100 & 77.78 \\
\hline MV & Mean & 95 & 93.33 & 86.67 & 100 & 77.78 \\
\hline & 2 & 76.92 & 71.43 & 100 & 100 & 89.47 \\
& 3 & 92.31 & 78.57 & 87.50 & 95.08 & 94.74 \\
& 4 & 76.92 & 92.86 & 100 & 86.89 & 84.21 \\
\hline & Mean & 80.77 & 83.93 & 95.31 & 94.67 & 89.47 \\
\hline
\end{tabular}

The rate of correct classification using the CBR technique is summarized in Table 3 . This table shows a significant increase in the rate of classification with respect to that obtained with the $T^{2}-Q$ statistics criterion.

A profound look at Fig. 4 and 5 illuminates that the classification rate along the circle radius axis after 1250 never changes, while for the next axis, number of nearest neighbors, it varies from beginning up to end. This fact can be easily interpreted based the sorted distances in the $T^{2}-Q$ plane. The majority of those distances are less than 1000 which mean that when we increase the radius of the circle in the $T^{2}-Q$ plane, most of them come into the considered region. In this case, the more impressive factor on classification rate is the number of neighbors as 
depicted in these figures. First, using auto-scaling method paves the way for comparing the processed data in one scale while they are originally quite different in terms of magnitude. Second, computing distances in $T^{2}-Q$ plane is the best alternative for taking into account similarities and differences between HV and MV sags in our classification. The supporting reason is arising from this fact that $T^{2}$ and $Q$ are entailing the information of all scores and not captured variations for different batches. Choosing each of $T^{2}$ or $Q$ alone will substantially lower the classification rate. Third, Fig. 4 and 5 make us sure that the taken values for $K$ and threshold result in the highest possible rate of classification under the mentioned circumstance.

\section{CONCLUSIONS AND FUTURE WORKS}

In this paper, we developed a hybrid method for classification of electrical sags in two classes: High Voltage (HV) and Medium Voltage (MV). The proposed method is a distance-based classifier which has been applied on the principal component space (MPCA projection) instead of comparing original original waveforms in the time domain directly. Origin of new sags is determined by projecting it into two MPCA models (HV and MV) and its class is determined based on the class of its neighbor majority in a bounded region on the $T^{2}-Q$ plane. Majority and region concepts build up an optimization problem which is solved numerically resulting in the maximum classification rate of electrical sags.

The capability of the proposed method was examined with the real HV and MV data sets recorded in five distribution substations. Results obtained with these data were significantly better than those obtained with traditional MPCA methods. Apparently, the performance of this hybrid classifier can be substantially improved through calculations of the distances in different spaces and comparison of them. Furthermore, the traditional statistical method can be used as a complement beside the proposed method leading to a perfect classification rate. These issues with more details will be addressed in next works.

The results obtained with this method also improve previous classification ratios obtained by the authors by extracting temporal and phasorial features from waveforms and using them as input of a classifier [12]. A possible explanation can be based on the capability of M-PCA to retain the information of the original waveform and the fact that these new features (scores) are independent. Independence between features is assumed by classifiers based on the distance criteria as CBR but not this is not always true.

\section{REFERENCES}

[1] A. Aamodt, E. Plaza Case-based reasoning: foundamental issues, methodological variations, and system approaches Artificial intelligence communications, 1:39-59, 1994.

[2] M.H.J. Bollen Understanding Power Quality problems, IEEE Press, New York, 2000.

[3] B.M. Wise, N.B. Gallagher, S. Watts, D.D. White Jr, and G.G. Barna. A comparison of PCA, Multiway PCA, trilinear decomposition and parallel factor analysis for fault detection in a semiconductor etch process, Journal of Chemometrics,13,1999.

[4] F. Gehhardt, A.V.W. Grather, B.S. Belz, Reasoning with complex cases, Kluwer International Series in Engineering and Computer Science, Boston, 1997.

[5] R.A. Johnson, D.W. Wichern, Applied Multiway Statistical Analysis, ISBN 0-13-041807-2, third edition edition, 1992.

[6] Theodora Kourti, Process analysis and abnormal situation detection: From theory to practice, IEEE Control Systems Magazine, 22(5), pp. 10-25, oct 2002.

[7] H. Langseth, A. Aamodt, O.M. Winnem, Learning retrieval knowledge from data, IJCAI99 Workshop ML-5: Automating the construction of case-based reasoning, Anand and Aha Eds., pp. 77-82, 1999.

[8] C.H. Lee, Y.J. Wang, W.L. Huang, A Literature Survey of Wavelets in Power Engineering Applications, Proc. Natl. Sci. Counc. ROC(A), Vol. 24, No. 4, 2000. pp. 249-258.

[9] J. F. MacGregor, editor, Multivariate Statistical Approaches to Fault Detection and Isolation, 5th IFAC SAFEPROCESS, 2003.

[10] J. Melendez, J. Colomer, J.L. Rosa, Expert supervision based on cases, Proceedings. 2001 8th IEEE International Conference on Emerging Technologies and Factory Automation, pp. 431-440, 2001.

[11] O. Poisson, P. Rional, M. Meunier, Detection and measurement of power quality disturbances using wavelets transform. IEEE TPD, Vol. 15, (3), 2000

[12] J. Melendez, J.J. Mora, D. Llanos, J. Colomer, J. Sanchez, Classification of Sags Measures in a Distribution Substation Based On Qualitative and Quantitative Temporal Attributes, 17th International Conference on Electricity Distribution,Barcelona, 2003.

[13] Paul Nomikos and John F. MacGregor, Monitoring batch processes using multiway principal component analysis, AIChE, 40(8):1361-1375, aug 1994.

[14] Giorgio Skonieczny and Benedetto Torrisi, editors, PCA, PLS and ANN: diversification of the Italian financial system, Third International Symposium on PLS and Related Methods, 2003.

[15] Styvaktakis, E. Bollen, M.H.J. and Gu, Y.H. Classification of power system events: voltage dips, Ninth International Conference on Harmonics and Quality of Power, Vol.2., pp. 745 -750, 2000.

[16] V. Venkatasubramanian, R. Rengaswamy, K. Yin, S.N. Kavuri: A review of process fault detection and diagnosis Part III: Process history based methods, Computers and Chemical Engineering Vol. 27, 327346, 2003

[17] I. Watson, F. Marir, Case-Based Reasoning: A Review, The knowledge of Engineering Review, Vol. 9, No. 4, 1994.

[18] Z. Wen, J. Crossman, J. Cardillo, Y.L. Murphey, Case Base Reasoning in Vehicle Fault Diagnostics, Proceedings of the International Joint Conference on Neural Networks, pp. 2679-84, 2003.

[19] B.M. Wise, N.S. Gallagher, The process chemometrics approach to process monitoring and fault detection, J. Proc. Cont., Vol. 6, No. 6, pp. 329-348, 1996.

[20] S. Yoon, J.F. MacGregor, Statistical and causal model-based approaches to fault detection and isolation, AIChE Journal, 46, pp. 1813-1824, 2000. 